\title{
"Seeing" iPSCs in the Retina During Retinal Repair in Diabetic Retinopathy
}

Sergio Li Calzi ${ }^{1}$, Cristiano P Vieira ${ }^{1}$, Dibyendu Chakraborty ${ }^{1}$, Ashwath Jayagopal ${ }^{2}$, Mervin C. Yoder ${ }^{3}$, Maria B. Grant ${ }^{1}$.

1. Department of Ophthalmology, University of Alabama, Birmingham, AL, USA.

2. Pharma Research and Early Development, Roche Innovation Center Basel, F. Hoffmann-La Roche, Ltd., Basel, Switzerland.

${ }^{3 .}$ Department of Pediatrics, Indiana University, Indianapolis, IN, USA.

In chronic diabetes, vascular reparative mechanism can be lost resulting in development of microvascular complications (MVC), such as diabetic retinopathy (DR). Reparative cells circulate in the peripheral blood and can either be derived from the bone marrow or form the vascular wall itself. The reparative function of progenitor cells can be assessed by in vitro assay as well as in vivo assays. Previously, we showed that vascular wall-derived progenitor cells, endothelial colony forming cells (ECFCs), are depleted in diabetics with MVC [1]. Bone marrow-derived progenitor cells, $\mathrm{CD} 45^{+} \mathrm{CD} 34^{+}$cells, are also dysfunctional in diabetics with MVC [2, 3]. Human Inducible Pluripotent Stem cells (hiPSCs) represent a novel approach for tissue repair and allow for limitless production of autologous cells. hiPSC-derived ECFCs displayed the ability to form functional and durable blood vessels in vivo and conferred therapeutic revascularization by connecting with and remaining integrated with host rodent vessels long term [4].

We asked if a mesoderm subset (SSEA5 ${ }^{-} \mathrm{KNA}^{+}$cells) generated from hiPSCs could give rise to endothelial cells and pericytes in three models of retinal injury, the retinopathy of prematurity model, the retinal ischemia/reperfusion ( $\mathrm{I} / \mathrm{R})$ injury model and a diabetic model, $\mathrm{db} / \mathrm{db}$ mice. We also tested whether the addition of iPSC- derived $\mathrm{CD} 45^{+} \mathrm{CD} 34^{+}$cells further enhanced the ability of SSEA5 ${ }^{-} \mathrm{KNA}^{+}$cells to promote retinal repair.

SSEA5 $-\mathrm{KNA}^{+}$cells in varying concentrations (alone or in combination with $\mathrm{CD} 45^{+} \mathrm{CD} 34^{+}$cells) were injected intravitreally into mice and the ability of SSEA5 ${ }^{-} \mathrm{KNA}^{+}$cells to home to areas of injury and form endothelial cells and pericytes was assessed by immunohistochemistry and analyzed by confocal microscopy using retinal flat mounts prepared from either ROP pups (Figure), I/R retinas or diabetic retinas. HYPOX-4, a fluorescence-imaging probe capable of detecting retinal-hypoxia in living animals was also used to assess improvements in retinal oxygenation in each model. Predicted patterns of retinal hypoxia were imaged by HYPOX-4 dependent fluorescence activity in each model prior to iPSC injection and then at regular intervals followed injection of iPSCs. In vivo fundus imaging was performed using the Micron IV Retinal Imaging Microscope. At completion of each study, mice were sacrificed and retinal flat mounts were prepared to assess localization and incorporation of human cells in the murine vasculature by confocal microscopy.

$\mathrm{SSEA}^{-} \mathrm{KNA}^{+}$cells were found to successfully generate both endothelial cells and pericytes in three different models of retinal injury. The coadministration of hiPSCs derived $\mathrm{CD} 34^{+} \mathrm{CD} 45^{+}$with SSEA5$\mathrm{KNA}^{+}$cells resulted in the enhanced survival, function and reparative ability of the SSEA5 ${ }^{-} \mathrm{KNA}^{+}$cells in each model tested. This beneficial effect was mediated by reducing retinal oxidative stress and inflammation.

In summary, current interventions to foster normal vascular remodeling and restoration of blood flow to the ischemic and injured retina are limited. Our studies combined conventional microscopy of retinal tissue with advanced in vivo functional imaging procedures to support the idea that hiPSCs represent a 
novel tool to facilitate retinal vascular restoration. We also found that the combinations of two iPSC populations worked synergistically to optimize retinal repair [5].

\section{References:}

[1] Jarajapu YP et al, PLoS One 9(4) (2014).

[2] Bhatwadekar AD, et al, Vision Res. 139 (2017), p. 211.

[3] Hazra S, et al, Diabetologia 56(3) (2013), p. 644.

[4] Prasain N, Nat Biotechnol. 32(11) (2014), p. 1151.

[5] Support: NIH grants: R01EY0126001, R01EY007739, R01HL110170, R01DK090730. Research to Prevent Blindness Unrestricted grant awarded to the Department of Ophthalmology.

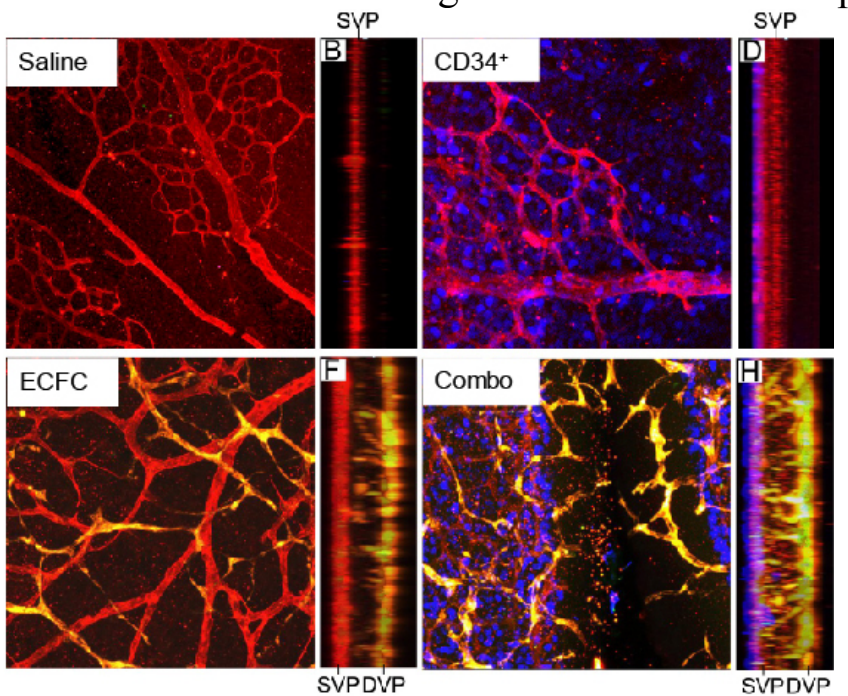

Figure 1. ECFCs stimulate the development of the Deep Vascular Plexus (DVP) in the developing retina exposed to hyperoxia. Representative z-stack projections of confocal images of flat mounted retinas from OIR pups injected on P5 and euthanized on P12. Panels $\mathrm{B}, \mathrm{D}, \mathrm{F} \& \mathrm{H}$ are 3D-projections of the corresponding left panel turned on edge showing the cross section of the retina (vitreous, left side; choroid, right side). CD34 ${ }^{+}$cells (blue). Blood vessels are stained with collagen IV Ab (red). ECFCs express GFP (green). ECFC incorporated into blood vessels appear yellow. SVP = superficial vascular plexus. DVP = deep vascular plexus. 